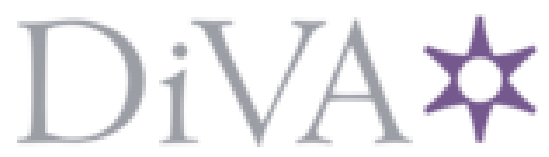

http://www.diva-portal.org

This is the published version of a paper presented at 12th World Conference on Mobile and Contextual Learning (mLearn 2013).

Citation for the original published paper:

Sotsenko, A., Jansen, M., Milrad, M. (2013)

About the Contextualization of Learning Objects in Mobile Learning Settings.

In: QScience Proceedings: Vol. 2013, 12th World Conference on Mobile and Contextual Learning (mLearn 2013) (pp. 67-70). Qatar

QScience Proceedings

http://dx.doi.org/10.5339/qproc.2013.mlearn.11

N.B. When citing this work, cite the original published paper.

Permanent link to this version:

http://urn.kb.se/resolve?urn=urn:nbn:se:Inu:diva-31412 


\title{
About the Contextualization of Learning Objects in Mobile Learning Settings
}

\author{
Alisa Sotsenko \\ Linnaeus University \\ Växjö, Sweden \\ alisasotsenko@gmail.com
}

\author{
Marc Jansen \\ University of Applied Sciences \\ Ruhr West \\ Bottrop, Germany \\ marc.jansen@hs-ruhrwest.de
}

\author{
Marcelo Milrad \\ Linnaeus University \\ Växjö, Sweden \\ marcelo.milrad@lnu.se
}

\begin{abstract}
In recent years, many efforts have been undertaken in order to design and deploy learning activities that make use of modern mobile devices, like smartphones and tablet PC's. Hence, new possibilities for supporting these so-called mobile learning scenarios have risen. One of the major benefits of these kinds of learning scenarios is the possibility of a learner to have access to learning content independent of time and place and therefore, enabling learners to learn in very different situations. In order to support learning across different settings, this paper discusses an approach that allows identifying a best fitting format of a Learning Object (LO) with respect to the current situation of the learner. This approach allows to delivering learning content in a format that may suit the current context of the learner and therefore, it enables seamless learning.
\end{abstract}

\section{Author Keywords}

Contextualized support for mobile learners, Mobile Learning Objects, multidimensional vector space model, similarity metrics

\section{INTRODUCTION}

The massive adoption of mobile technologies combined with access to content in a wide variety of settings allows learners to experience new learning situations beyond the borders of the classroom. Cross-contextual learning can enable a continuous learning experience across different settings, such as home-school, or workplace-college. There are different approaches to provide contextual support for learners in their current learning situations. All of them have some limitations and require improvements and/or extensions. For instance, using only context information about the learner's location may be not enough, while the noise level of the location (being indoors or outdoors) might be very important to keep the concentration of the learner at a high level. Moreover, each learner has different interests and ways of learning (Wang \& Karlström, 2012). Limitations of mobile devices such as small screen size, keyboard, etc. provide another challenge to face (Giemza \& Jansen, 2011). In order to address the above mentioned problems, we provide an approach that suggests the best suitable format of learning objects (LO) that best-fit the current context of the learner.

The paper is organized as follows. Section two gives a short overview of the advantages provided by the contextualization of learners in different learning settings. Additionally, it describes which contextual information can be used in order to achieve this contextualization. After that, the next section discusses different approaches for recommender systems and suggests a multidimensional vector space model (MVSM) that can be used to provide contextualized support to learners. Section four describes how different formats of LOs can be used in different contexts by using this multidimensional vector space model. Moreover, we discuss different similarity metrics. Finally, section five describes in detail an algorithm that allows defining the best-fit format of certain LOs. Section six presents an outlook and ideas with regard to our future work.

\section{RELATED WORK}

The provision of contextualized support to mobile learners could be of great use in different learning scenarios. For instance, Cui (2005) describes a scenario to improve English knowledge of Chinese students by providing a mobile intelligent tutoring system that includes individual learner's knowledge and features of the learner's current location. In particular, this paper states that the current location may affect the concentration of the learner and the amount of available time to study. In another scenario, Gicquel \& Lenne (2012) extend an informal learning scenario that takes place during museum visits by providing contextualized support to the visitor. Moreover, the application suggests other arts or styles, etc. with respect to the current location and the interests of the visitor. In this particular scenario, the benefit of contextualization is to provide guided support to the visitor. Contextualized media for learning is an approach used by De Jong et al., (2008) that utilizes different media formats for event notification, documentation and communication between learners across different learning contexts. Several benefits can be identified while summarizing the formerly described learning scenarios for supporting the contextualization of learners; i) an increased motivation to study any time and any place, ii) they provide opportunities to study fast and relevant to learners current location and iii) provide personalized learning experience in real life situations.

The context of the learner may include different pieces of information. As described in Brown, (2010), the context of a learner can include information about the environment he/she is currently located in (environment context), his/her personal context, e.g. interests, language, learning courses, available time to study (personal context) and/or context 
information about the activity the learner is currently involved in (activity context), etc. (Kurti, 2008). There is already a substantial body of research that describes context information and the different associated dimensions. All those dimensions of contextual information can be acquired with the help of mobile devices and additional Web Services, e.g. the location of the learner can be obtained by the GPS sensor, the nearest place and further places of interest can additionally be retrieved, e.g., by using Web Services like the Google Place API, or to get fast and easy access to LOs by scanning barcodes, QR codes with camera on mobile device etc. After a literature review of this field, we organized the contextual information in a three dimensional space where each dimension contains sub-dimensions as shown in table 1.

\begin{tabular}{|c|c|c|}
\hline Environment context & Device context & Personal context \\
\hline Current location, & Battery charge, & Interests, \\
Nearest places, & Language, & Goals, \\
Weather, Direction, & Connection speed, & Subject matter/courses, \\
Noise level, & Kcreen size & Knowledge, \\
Movement (e.g. & & Available time to study \\
moving, sitting) & & Preferred representation type \\
Time and date & & \\
\hline
\end{tabular}

Table 1. Dimensions of contextual information

These dimensions provide the theoretical foundation that guide the development of an algorithm that allows defining the best-fit format of certain LOs for a given context. These ideas will be described in the following sections.

\section{DISCUSSION OF RECOMMENDER SYSTEMS}

One of the approaches for the integration of contextual information in learning scenarios is a personalized learning system with adaptation features related to LOs or learning activities. Adaptation to learning objects can be achieved by getting contextual information, e.g., from mobile sensors or web services, about the current context of the learner. Furthermore, LOs can be presented by different formats for the same type and content of the LO (e.g. .wav files to .mp3) or different styles for the same content of LO (e.g. font size, brightness, etc.) (Zervas et al., 2013). Such type of systems may suggest, e.g., LOs according to the user's information need, places, time and moment by using retrieval algorithms (e.g. ranking models such as vector space models, probabilistic models and clustering models) (Melucci, 2005). Yang et al., (2010) have proposed a context-driven content adaptation planner with respect to the user's environments in order to increase the performance of mobile web-based applications by using the Object Structure Model (OSM) combined with a JESS-enabled rule engine. Whereas adaptation of learning activities might be performed by considering, e.g., the recent subjects of study, learners interests, goals, tasks etc. For instance, the PEACOCK e-learning management system (Muntean \& Muntean, 2009) provides selection of rich learning content (e.g. multimedia, text, pictures) to users that best fit to their interests, goals, device properties, network connection, etc. Their results show an increased learning satisfaction by providing the best-fit learning content that matches the user's profile, device properties and network connection. Furthermore, sensors available on mobile devices (GPS, accelerometer, etc.), offer possibilities to provide flexible, exciting and interactive learning environments without the intervention of a teacher. For instance, Karavirta \& Hakulinen (2012) suggest learning of algorithms and data structures by playing games while using the accelerometer of a mobile device. All these systems have common goals, such as to improve the learner's satisfaction and increase interests and motivation while supporting learning across different settings and time.

According to Sampson \& Zervas (2013) the process of content adaptation consists the following phases: detection of the learners' mobile context dimensions, evaluation of these context dimensions by the content adaptation mechanism (e.g., content transformation, content filtering, content navigation, etc.) and finally the presentation of the adapted content to the learner. We assume that the learning activity and goals are pre-defined. Thus, one of the ways to support learning at any place and any time can be achieved by adapting the learning objects to the learner's context. For instance, the system suggests the format of the LO to the learner based on his/her current context in order to provide better suited formats with respect to the current context of the learner. One of the possible approaches to design a valid context information mechanism is a multi-dimensional vector space model (MVSM). Let's assume that each learning resource might be available in different formats (e.g., .pdf, .mp3, etc.). Then, each point in the MVSM represents a certain format of a certain LO. The learner should be satisfied of the format of LO in different learning scenarios. Thus, the format of the learning resource could be retrieved automatically that most likely fits best to the current context of the learner. Here, the requirements we concentrated on in order to provide the format of LO successfully, are to find formats that do support the learner in his current context, takes benefit of the current context or, at least, does not remove the learners concentration from other important issues. Therefore, this model can be used to identify an appropriate format of a LO by measuring the similarity between two different vectors: the vector describing the current context of the learner and another vector that describes each of the available LO. Based on previous work on recommender systems discussed above and in (Ricci et al., 2011), the LO that has the closest distance to the point of the current context of the learner in the MVSM, will most likely be the LO that fits best. 


\section{DESCRIPTION OF THE METHOD REPRESENTATING CONTEXTUAL INFORMATION IN MVSM}

The MVSM can be used for modeling context information as described in the previous section. In our model, three main context dimensions (environment, device context and personal) are included in one space in which the current context of the learner is represented by a distinguished point. Here, each vector provides information about the environment (place, level of noise, statement, etc.), personal interests (subject, interests, language etc.) and the device that the learner currently uses (battery charge, device screen, internet connection, etc.). The information encapsulated in the vectors represents the current context of the learner.

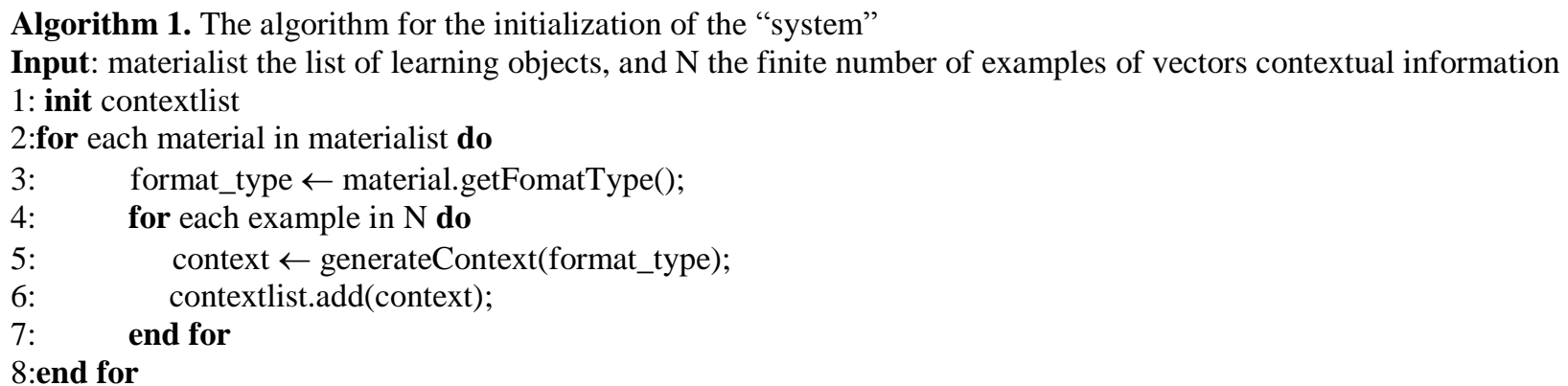

\section{8:end for}

For each format type of LO the different contextual information are generated by the generateContext(format_type) method. For instance, for the file type .pdf the environment context (current place - library, noise level - $15 \mathrm{~dB}$, movement - false, time - 14:00, etc. ), device context(battery charge - 80\%, language - English, connection speed $2 \mathrm{Mb} / \mathrm{s}$ ) and personal context (available time -2 hours, subject -Web Services, Preferred representation type- audio) are integrated in the environment.

Similarity measurements can be used in order to define the suggested format type of the learning object for the current context. The similarity between vectors can be calculated by using different metrics. Each dimension of contextual information can be represented with different data types and range of values. For instance, the temperature, noise level, battery charge and connection speed might be represented as decimal type while the movement might be represented as boolean type (e.g. true - moving, false - not moving). For the decimal values, the simple Euclidean distance can be used to measure the similarity between the current weather and a set of defined weathers in MVSM, etc. For instance, the Euclidean distance measures the similarity between two vectors of $n$ dimensions by calculating the distance between their components. The value of distance $d$ shows how much the one object differs from another one e.g., how much the current noise level in the café differs from the noise level in the library or university, etc.

Another metric that can be used with decimal values is the cosine similarity. This metric can be used to define the most similar vector of contextual information that represents the format of LO by calculating the angel between the vector of the current contextual information with decimal data types and examples generated from Algorithm 1. Moreover, this metric allows identifying the orientation of learners in different learning scenarios, e.g. if the cosine similarity is 1 then vectors of contextual information have the same orientation.

The environment context information about moving/standing, outdoor/indoor, etc. can be represented as boolean typed values. Here, the Jaccard metric for binary objects might be better suited than the cosine metric or Euclidean distance. Because measuring the overlap between sets of binary objects will provide more meaningful results than calculating the linear distance or cosine metric. In our case Jaccard similarity is defined as the size of the intersection of the context information described by boolean values (here, intersection means the components that are equal in both vectors) divided by the size of the union (here, all components described by boolean values) of the corresponding vector. The higher the Jaccard index is for two vectors, the more equal these two vectors are.

As discussed above, the Euclidean distance gives us the absolute value for the distance between two objects; hence we are interested in similarity between the same contextual information not in similarity of the differences of the contextual information (e.g. learner sitting in the café, we are interested to define a similar place to the café not the nearest place to the café,). The cosine similarity shows how similar or "close" one piece of contextual information is to another one. By summarizing the discussion from above, the different metrics could be used for different data types of contextual information; thus, in our approach we combined cosine and Jaccard metrics in order to match the current context of learners to format types of LO.

\section{IMPLEMENTATION}

In order to define the suggested format type of a LO for the current context of the learner, we represented his/her current context information in the proposed MVSM. Each component of this context information vector is in itself multidimensional, thus we define a sub-vector space for environment, device and personal context. Here are vectors that contain information about the current environment, device and personal context and $e_{1}, e_{2}, e_{3}, \ldots e_{k}$, $d_{1}, d_{2}, \ldots d_{m}, p_{1}, p_{2}, \ldots p_{l}$ are vectors representing formats of the LO in the MVSM. For each $e_{k}, d_{m}, p_{l}$ we measure the distance to vector $e^{\prime}, d^{\prime}, p^{\prime}$ by calculating cosine angel $\cos _{e}\left(e_{k}, e^{\prime}\right), \cos _{d}\left(d_{m}, d^{\prime}\right), \cos _{p}\left(p_{l}, p^{\prime}\right)$ for context information and Jaccard index $J_{e}\left(e_{k}, e^{\prime}\right), J_{d}\left(d_{m}, d^{\prime}\right), J_{p}\left(p_{l}, p^{\prime}\right)$ for contextual information that has Boolean data type. After that, we 
will have the two dimensional similarity vectors $\operatorname{sim}_{e}\left(\cos _{e}, J_{e}\right), \operatorname{sim}_{d}\left(\cos _{d}, J_{d}\right), \operatorname{sim}_{p}\left(\cos _{p}, J_{p}\right)$ for the environment, device and personal context. Then, the final format is defined by the vector in MVSM that has minimal absolute value $d_{\min }\left(\operatorname{sim}_{e}, \operatorname{sim}_{d}, \operatorname{sim}_{p}\right)$ of all similarity vectors. This allows implementing the algorithm below.

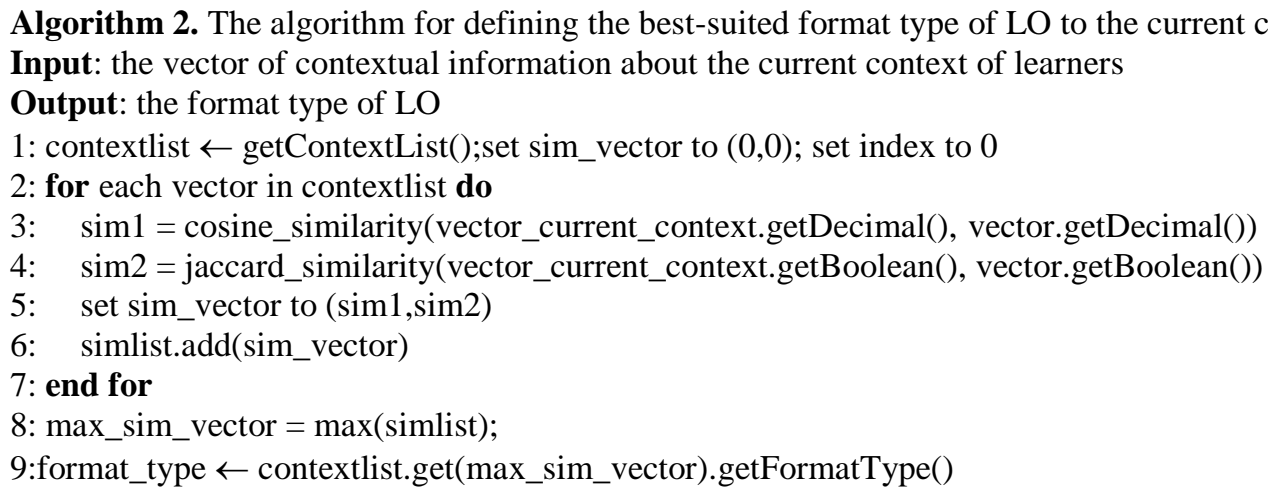

\section{OUTLOOK AND FUTURE WORK}

This paper proposes an approach for providing recommendations with regard to the format type of LO to be used with respect to the learner's current context. We presented a model for describing contextual information represented as a multidimensional vector space model that serves to match the format type of LO. We combined the cosine and Jaccard metric similarity to define the best-fit format of LO. Future plans include the deployment and evaluation of the presented approach. Here, we plan to implement the suggested approach in different mobile learning scenarios and with different groups of learners. Possible benefits of this approach may result in a cross-contextual learning process that becomes more personalized and adaptive, thus supporting seamless learning. Moreover, it may also result in improved motivation by offering LOs with respect to the learner knowledge and personal interests.

\section{REFERENCES}

Brown, E., Börner, D., Sharples, M., Glahn, C., de Jong, T., \& Specht, M. (2010). Location-based and contextual mobile learning. A STELLAR Small-Scale Study.

Cui, Y., \& Bull, S. (2005). Context and learner modeling for the mobile foreign language learner. System, 33 (2), (pp. 353-367).

De Jong, T., Specht, M., \& Koper, R. (2008). Contextualised Media for Learning. Journal of Educational Technology \& Society, 11(2), (pp. 41-53).

Gicquel, P.Y., Lenne, D. (2012). Using Semantic Similarities to Instrument Informal Learning Activities in Ubiquitous Environments. In Proc. of the 2012 IEEE 12th International Conference on Advanced Learning Technologies, (pp. 618-620).

Giemza, A., \& Jansen, M. (2011). An Architectural Approach to Support Multi-Device Learning Environments. In Proc. of the IADIS International Conference on Mobile Learning. Avila, Spain, (pp. 319-321)

Karavirta, V., \& Hakulinen, L. (2012). Educational Accelerometer Games for Computer Science. In Workshop Proc. CEUR 2012, (pp. 216-219).

Kurti, A. (2008). Context modeling to support the design of mobile learning. In Proc. of the 5th international conference on Soft computing as transdisciplinary science and technology, (pp. 536-541).

Melucci, M. (2005). Context modeling and discovery using vector space bases. In Proc. of the 14th ACM international conference on Information and knowledge management, (pp. 808-815).

Muntean, C. H., \& Muntean, G.-M. (2009). Open corpus architecture for personalized ubiquitous e-learning. Personal Ubiquitous Comput., 13(3), (pp.197-205)

Ricci, F., Rokach, L., \& Shapira, B. (2011). Introduction to recommender systems handbook. In Recommender Systems Handbook, (pp. 1-35). Springer US.

Wang, J., \& Karlström, P. (2012). Mobility and Multi-modality an Exploratory Study of Tablet Use in Interaction Design Learning. In Proc CEUR 2012, (pp. 276-279).

Yang, S. J. H., Zhang, J., Huang, J. J. S., \& Tsai, J. J. P. (2010). Using Description Logics for the Provision of Context-Driven Content Adaptation Services. Int. J. Syst. Serv.-Oriented Eng., 1(1), (pp. 96-129)

Zervas, P., Sampson, D., Gomez, S. \& Fabregat, R., (2013). Designing Tools for Context-Aware Mobile Educational Content Adaptation. Ubiquitous and Mobile Informal and Formal Learning in Digital Age, (pp. 3-18), Springer.

Zervas, P., Sampson, D., (2013). Context-Aware Adaptive and Personalised Mobile Learning Systems. Ubiquitous and Mobile Informal and Formal Learning in Digital Ag,, (pp. 37-48), Springer. 